\title{
In-depth Profiling of MvfR-Regulated Small Molecules in Pseudomonas aeruginosa after Quorum Sensing Inhibitor Treatment
}

\begin{abstract}
Giuseppe Allegretta ${ }^{1}$, Christine K. Maurer ${ }^{1}$, Jens Eberhard ${ }^{1}$, Damien Maura ${ }^{2,3,4}$, Rolf W. Hartmann ${ }^{1,5 *}$, Laurence Rahme ${ }^{2,3,4 *}$ and Martin Empting ${ }^{1 *}$
\end{abstract}

${ }^{1}$ Department of Drug Design and Optimization, Helmholtz Institute for Pharmaceutical Research Saarland, Saarbrücken, Germany, ${ }^{2}$ Department of Surgery and Department of Microbiology and Immunobiology, Harvard Medical School, Boston, MA, United States, ${ }^{3}$ Department of Surgery, Center for Surgery, Innovation and Bioengineering, Massachusetts General Hospital, Boston, MA, United States, ${ }^{4}$ Shriners Hospitals for Children, Boston, MA, United States, ${ }^{5}$ Pharmaceutical and Medicinal Chemistry, Saarland University, Saarbrücken, Germany

\section{OPEN ACCESS}

Edited by:

Dimitris G. Hatzinikolaou, National and Kapodistrian University

of Athens, Greece

Reviewed by:

Sotirios Vasileiadis,

University of Thessaly, Greece

Justin Joseph Donato,

University of St. Thomas,

United States

*Correspondence:

Martin Empting

martin.empting@helmholtz-hzi.de

Laurence Rahme

rahme@molbio.mgh.harvard.edu

Rolf W. Hartmann

rolf.hartmann@helmholtz-hzi.de

Specialty section:

This article was submitted to

Systems Microbiology,

a section of the journal

Frontiers in Microbiology

Received: 05 March 2017 Accepted: 08 May 2017

Published: 24 May 2017

Citation:

Allegretta G, Maurer CK, Eberhard J, Maura D, Hartmann RW, Rahme L and Empting $M$ (2017) In-depth Profiling of MvfR-Regulated Small

Molecules in Pseudomonas aeruginosa after Quorum Sensing Inhibitor Treatment.

Front. Microbiol. 8:924. doi: 10.3389/fmicb.2017.00924
Pseudomonas aeruginosa is a Gram-negative bacterium, which causes opportunistic infections in immuno-compromised individuals. Due to its multiple resistances toward antibiotics, the development of new drugs is required. Interfering with Quorum Sensing (QS), a cell-to-cell communication system, has shown to be highly efficient in reducing $P$. aeruginosa pathogenicity. One of its QS systems employs Pseudomonas Quinolone Signal (PQS) and 4-hydroxy-2-heptylquinoline (HHQ) as signal molecules. Both activate the transcriptional regulator MvfR (Multiple Virulence Factor Regulator), also called PqsR, driving the production of QS molecules as well as toxins and biofilm formation. The aim of this work was to elucidate the effects of QS inhibitors (QSIs), such as MvfR antagonists and PqsBC inhibitors, on the biosynthesis of the MvfR-regulated small molecules 2'-aminoacetophenone (2-AA), dihydroxyquinoline (DHQ), HHQ, PQS, and 4-hydroxy-2-heptylquinoline- $N$-oxide (HQNO). The employed synthetic MvfR antagonist fully inhibited pqs small molecule formation showing expected sigmoidal dose-response curves for 2-AA, HQNO, HHQ and PQS. Surprisingly, DHQ levels were enhanced at lower antagonist concentrations followed by a full suppression at higher QSI amounts. This particular bi-phasic profile hinted at the accumulation of a biosynthetic intermediate resulting in the observed overproduction of the shunt product $\mathrm{DHQ}$. Additionally, investigations on PqsBC inhibitors showed a reduction of MvfR natural ligands, while increased 2-AA, DHQ and HQNO levels compared to the untreated cells were detected. Moreover, PqsBC inhibitors did not show any significant effect in PA14 pqsC mutant demonstrating their target selectivity. As 2-AA is important for antibacterial tolerance, the QSIs were evaluated in their capability to attenuate persistence. Indeed, persister cells were reduced along with 2-AA inhibition resulting from MvfR antagonism, but not from PqsBC inhibition. In conclusion, antagonizing MvfR using a dosage capable of fully suppressing this QS system will lead to a favorable therapeutic outcome as DHQ overproduction is avoided and bacterial persistence is reduced.

Keywords: Pseudomonas aeruginosa, quinolones, 2'-aminoacetophenone, dihydroxyquinoline, persistence, Quorum Sensing Inhibitors, MvfR 


\section{INTRODUCTION}

Pseudomonas aeruginosa is a ubiquitous Gram-negative bacterium able to cause severe chronic infections in immuno-compromised patients, for example in people affected by cystic fibrosis (Gómez and Prince, 2007) or thermally injured individuals (Tredget et al., 2004). The eradication of this pathogen with antibiotic treatments is becoming more and more difficult because of its intrinsic and acquired resistance (Hancock and Speert, 2000; Aloush et al., 2006) and tolerance (Mulcahy et al., 2010) toward these drugs. A new promising strategy for treating $P$. aeruginosa infections is blocking its pathogenicity without killing the bacteria targeting a cell-to-cell communication system called Quorum Sensing (QS) (Hurley et al., 2012).

This bacterium employs four QS systems interconnected to each other, namely las, iqs, $p q s$, and $r h l$, for regulating the expression of several toxins needed for adjusting its metabolism and virulence during the course of infection (Lee and Zhang, 2015). The $p q s$ QS system is selectively expressed by $P$. aeruginosa and utilizes the signal molecule Pseudomonas Quinolone Signal (PQS) and its precursor 4-hydroxy-2-heptylquinoline (HHQ) for activating the transcriptional regulator MvfR (Multiple Virulence Factor Regulator), also called PqsR. This protein induces the production of different toxins, such as lectins, pyocyanin, and hydrogen cyanide. It also regulates the expression of enzymes needed for the biosynthesis of its natural ligands encoded by the pqsABCDE operon (Xiao et al., 2006) and has been shown to be essential for persister cells development (Que et al., 2013).

Briefly, the synthesis of HHQ and PQS starts with the conversion of anthranilic acid (AA) into its Coenzyme A (CoA) thioester derivative by the action of CoA-ligase PqsA. The activated molecule is then condensed with malonyl-CoA by PqsD leading to the formation of $2^{\prime}$-aminobenzoylacetyl-CoA (2-ABA-CoA), which is subsequently hydrolyzed by the thioesterase PqsE or TesB into 2 '-aminobenzoylacetate (2-ABA) (Dulcey et al., 2013; Drees and Fetzner, 2015). This reactive intermediate is transformed into HHQ by the heterodimer PqsBC bearing an octanoyl chain (Dulcey et al., 2013). Finally, PqsH oxidizes HHQ into PQS (Schertzer et al., 2010) (Figure 1).

Furthermore, 2-ABA-CoA and 2-ABA are intermediates for the biosynthesis of other important metabolites. Actually, both compounds can cyclize leading to the formation of dihydroxyquinoline (DHQ), which has been shown to be fundamental in P. aeruginosa pathogenicity (Gruber et al., 2016), and in reducing the growth of epithelial cells (Zhang et al., 2008). Moreover, after decarboxylation, 2-ABA is converted into 2 '-aminoacetophenone (2-AA), an important signal molecule that coordinates the transition from acute to chronic infection and the development of persister cells (Kesarwani et al., 2011; Que et al., 2013). In addition, 2-ABA could be converted into its hydroxylamine form by the oxidase PqsL and, then, transformed into 4-hydroxy-2-heptylquinoline-N-oxide (HQNO) by the complex octanoyl-PqsBC (Dulcey et al., 2013). HQNO is essential for biofilm formation because it favors extracellular DNA release by programmed cell lyses of the bacteria (Hazan et al., 2016).
Among the potential targets for blocking the pqs system, we herein discuss the transcriptional regulator $\mathrm{MvfR}$ and the biosynthetic enzyme PqsBC. So far, a number of MvfR antagonists and PqsBC inhibitors have been developed that efficiently reduced $\mathrm{HHQ}$ and PQS production in P. aeruginosa (Zender et al., 2013; Lu et al., 2014a; Starkey et al., 2014). The aim of this work was to gather detailed information about the effects of these QS Inhibitors (QSIs) on the production of MvfR-related small molecules including 2-AA, DHQ, HQNO, HHQ, and PQS. Furthermore, we monitored the expression of the $p q s$ operon in a time-dependent manner upon treatment with the aforementioned QSIs.

Among the QS molecules, 2-AA was proven to be important in the development of $P$. aeruginosa persister cells (Que et al., 2013), which are individuals within the bacterial population characterized by having a reduced metabolism (Lewis, 2010). Due to their dormant state, antibiotic efficacy is severely impaired in this bacterial sub-group. Targeting persistence by blocking 2-AA production through QS inhibition was shown to be a promising strategy (Starkey et al., 2014). Consequently, an additional goal of the work was to quantify persister phenotype of $P$. aeruginosa after treatment with QSIs.

\section{MATERIALS AND METHODS}

\section{Chemicals and Growth Media}

The benzimidazole 1 and the nitrophenylmethanol 2 (Figure 2) were synthesized as described in literature (Rahme et al., 2012; Storz et al., 2014). $d_{4}$-HHQ was synthesized following the procedure of HHQ using $d_{5}$-aniline (Lu et al., 2012). The tricyclic 3 (Figure 2) and amitriptyline were purchased from ChemDiv (United States) and Alfa Aesar (Germany), respectively.

Water (Th. Geyer, Germany), acetonitrile (VWR, Germany) methanol (Sigma-Aldrich, United States) and formic acid (Fluka, United States) were LC-MS grade and used for HPLC-MS/MS experiments.

Yeast extract (Fluka, Germany), sodium chloride (VWR, Germany) and peptone from casein (Merck, Germany) were used for the preparation of Luria Bertani (LB) broth needed for performing the quantification experiments of $p q s$-related signal molecules. Ready-made mixture of LB broth (Fisher, United States) and Tryptic Soy Broth (TSB) $1 \%[w / v]$ (BD, United States) were used for pqsA-GFP $\mathrm{ASV}$ and persister cells experiments.

\section{Bacterial Strains}

$P$. aeruginosa PA14 and its isogenic transposon mutants $p q s A$, $p q s C, p q s H$ and $p q s E$ kindly provided by Susanne Häussler (Twincore, Hannover, Germany) were used in the experiments for the quantification of MvfR-related small molecules with and without QSIs. P. aeruginosa PA14, its isogenic pqsBC transposon mutant, and its $m v f R$ single mutant were employed for performing persister cells assays. $P$. aeruginosa PA14 transformed with pAC37 carrying pqsA-GFP ${ }_{\text {ASV }}$ and gentamicin resistance cassette was used in $p q s A$ expression experiments. The bacterial strains were maintained at $-80^{\circ} \mathrm{C}$ in $25 \%[v / v]$ glycerol stocks. 
<smiles>Nc1ccccc1C(=O)O</smiles>

AA



$\mathrm{MCoA} \backslash \mathrm{PqsD}$<smiles>Nc1ccccc1C(=O)CC(=O)[Se]OC(=O)O</smiles>

2-ABA-CoA

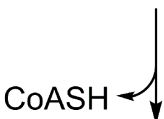<smiles>Oc1cc(O)c2ccccc2n1</smiles>

DHQ<smiles>O=C(O)CC(=O)c1ccccc1NO</smiles>

2-HABA<smiles>C1C[C@H]2C[C@@H](C2)[TeH4]1</smiles><smiles>NC1=CC=C2C=CC(=C1C(=O)CC(=O)O)C2[Pb]</smiles>

2-ABA<smiles>CC(C)[14CH3]</smiles><smiles>CC(=O)c1ccccc1N</smiles>



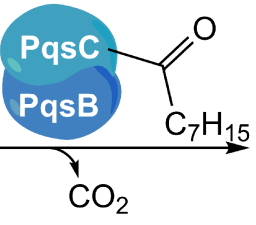

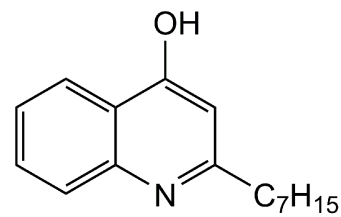

HHQ

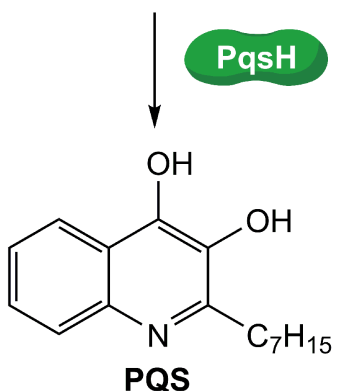

FIGURE 1 | Current model of the biosynthetic pathway of MvfR-related small molecules. AA, anthranilic acid; CoASH, Coenzyme A; MCoA, malonyl-CoA; 2-ABA-CoA, 2'-aminobenzoylacetyl-CoA; 2-ABA, 2'-aminobenzoylacetate; DHQ, dihydroxyquinoline; 2-AA, 2'-aminoacetophenone; 2-HABA,

2'-hydroxylaminobenzoylacetate; HHQ, 4-hydroxy-2-heptylquinoline; HQNO, 4-hydroxy-2-heptylquinoline-N-oxide; PQS, Pseudomonas Quinolone Signal.

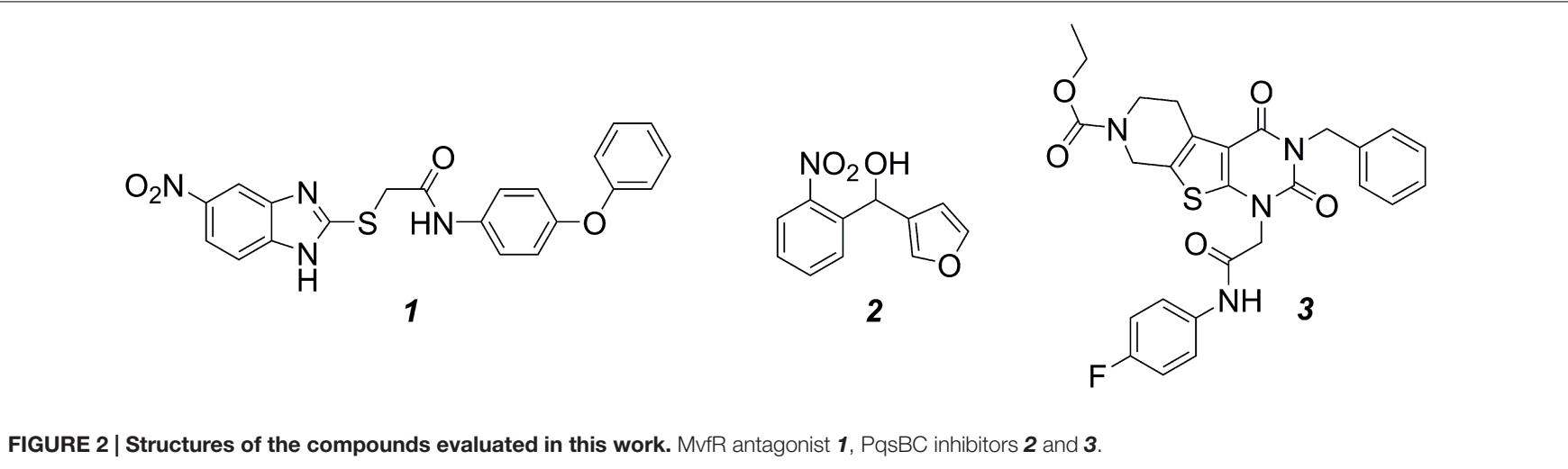

\section{MvfR-Related Small Molecule Quantification}

Pseudomonas aeruginosa strains were cultivated as previously described (Maurer et al., 2013). After letting PA14 strains grow for $6 \mathrm{~h}$ in $\mathrm{LB}$, an aliquot of bacterial culture was centrifuged for $10 \mathrm{~min}$ at $4835 \times \mathrm{g}$ and $25^{\circ} \mathrm{C}$ using a Rotina $380 \mathrm{R}$
Centrifuge (Hettich, Germany). The supernatant was discarded, the pellet was resuspended in fresh LB, and the bacterial cells were spun down using the previous centrifugation settings. After repeating the wash step a second time, the $\mathrm{OD}_{600}$ of the washed cells was measured using a BioPhotometer plus Spectrophotometer (Eppendorf, Germany) in order to prepare 
TABLE 1 | Effects of the MvfR antagonist 1 on production of MvfR-related small molecules in PA14 wt and PA14 pqsH mutant.

\begin{tabular}{|c|c|c|c|c|c|}
\hline Strains & $2-A A I_{50}[\mu M]$ & Maximal DHQ production [\%] & HQNO IC ${ }_{50}[\mu \mathrm{M}]$ & Signal molecules $^{\mathrm{a}} \mathrm{IC}_{50}[\mu \mathrm{M}]$ & Overall $I_{50}[\mu \mathrm{M}]$ \\
\hline PA14 wt & $1.3(1.1-1.2)^{\mathrm{b}}$ & $330 \pm 12^{\mathrm{c}} @ 1 \mu \mathrm{M}$ & $1.2(1.1-1.3)^{\mathrm{b}}$ & $1.1(1.0-1.2)^{\mathrm{b}}$ & $1.2(1.1-1.3)^{b}$ \\
\hline PA14 pqsH mutant & $0.19(0.16-0.22)^{b}$ & $304 \pm 2^{\mathrm{c}} @ 0.2 \mu \mathrm{M}$ & $0.27(0.25-0.30)^{b}$ & $0.27(0.25-0.30)^{b}$ & $0.32(0.30-0.34)^{b}$ \\
\hline
\end{tabular}

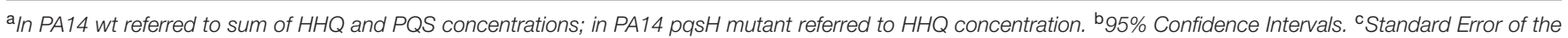
Mean intervals.

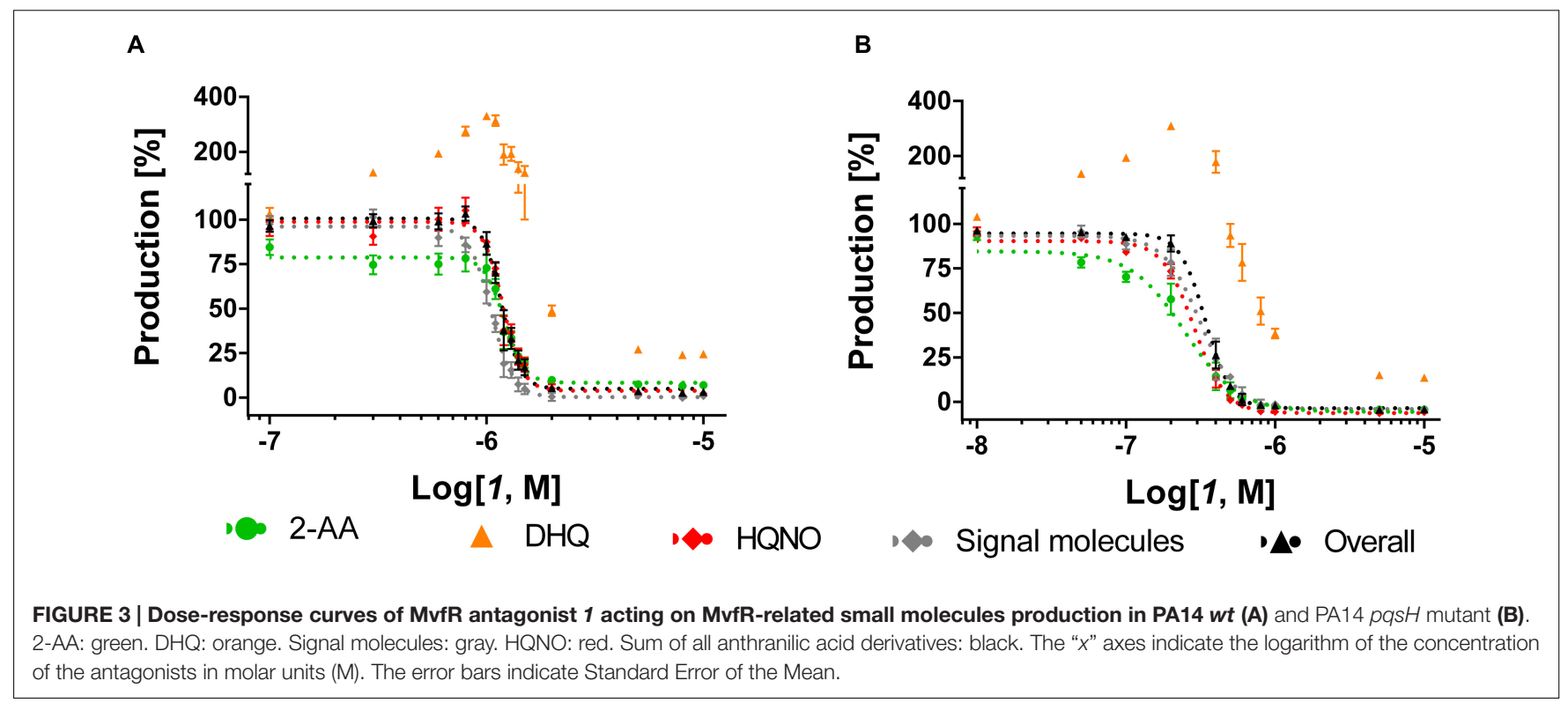

TABLE 2 | Effects of MvfR antagonist 1 on 2-AA and DHQ production in PA14 pqsC mutant with and without external addition of PQS.

\begin{tabular}{|c|c|c|c|c|}
\hline Compounds & Exogenous PQS $[\mu \mathrm{M}]$ & $2-A A \mid C_{50}[\mu M]$ & $\mathrm{DHQ} I \mathrm{C}_{50}[\mu \mathrm{M}]$ & $2-A A+D H Q I C_{50}[\mu \mathrm{M}]$ \\
\hline \multirow[t]{3}{*}{1} & 0 & $0.05(0.04-0.06)$ & $0.03(0.03-0.04)$ & $0.03(0.02-0.04)$ \\
\hline & 1 & $0.33(0.28-0.38)$ & $0.09(0.05-0.18)$ & $0.11(0.07-0.19)$ \\
\hline & 10 & $4.2(3.0-5.8)$ & $1.7(1.3-2.3)$ & $2.0(1.4-2.8)$ \\
\hline
\end{tabular}

The data are reported with 95\% Confidence Interval.

a final bacterial suspension with $\mathrm{OD}_{600}=0.02$ in LB. Aliquots of $1.5 \mathrm{~mL}$ were added in each well of a 24 -well Cellstar plate (Greiner Bio-One, Frickenhausen, Germany). Fifteen microliters of dimethyl sulfoxide (DMSO) or DMSO stock solutions of the target compound were added in each well. Triplicates of each condition were evaluated in every assay. The plates were incubated for $17 \mathrm{~h}$ at $37^{\circ} \mathrm{C}$ with $75 \%$ humidity and shaken at $200 \mathrm{rpm}$. The quantification of AA derivatives was performed following a previously described protocol (Lépine et al., 2003) with slight modifications. Seven hundred and fifty microliters of culture from each well were diluted with $750 \mu \mathrm{L}$ of Internal Standard (IS) stock solution in acetonitrile. The IS employed in experiments with PA14 wt and pqsC mutant was $d_{4}$-HHQ at a final concentration of $500 \mathrm{nM}$, while the experiments in PA14 $p q s H$ mutant required amitriptyline as IS at a final concentration of $1 \mu \mathrm{M}$. After mixing the diluted cell culture for $5 \mathrm{~min}$, the mixture was spun down for $15 \mathrm{~min}$ at $18,620 \times g$ and $15^{\circ} \mathrm{C}$ using a Mikro 220R Centrifuge (Hettich, Germany). One milliliter of supernatant was transferred in glass vial and analyzed with the Accela HPLC system (Thermo Fisher Scientific, Germany) coupled to a triple quadrupole mass spectrometer TSQ Quantum Access Max (Thermo Fisher Scientific Germany) equipped with an HESI-II source. Separation was achieved by a MachereyNagel Nucleodur $\mathrm{C}_{18}$ Pyramid column $(150 \mathrm{~mm} \times 2 \mathrm{~mm}$, $3 \mu \mathrm{m}$ ) heated at $40^{\circ} \mathrm{C}$. The mobile phase consisted of 10:90 $\mathrm{MeOH}: \mathrm{H}_{2} \mathrm{O}$ for $0.5 \mathrm{~min}$, followed by a linear gradient of $1.5 \mathrm{~min}$ for reaching $100 \% \mathrm{MeOH}$, which was kept constant for $1 \mathrm{~min}$. Then, the initial eluents composition was pumped for $2 \mathrm{~min}$. The flow rate employed was $600 \mu \mathrm{L} \cdot \mathrm{min}^{-1}$. A final concentration of $0.1 \%$ formic acid was present in the eluents. Compounds were ionized using heated electrospray ionization (hESI) in positive ion mode with the following parameters: spray voltage: $3500 \mathrm{~V}$; vaporizer temperature: $370^{\circ} \mathrm{C}$; sheath gas pressure (nitrogen): 35 units; auxiliary gas pressure (nitrogen): 30 units; skimmer offset voltage: $0 \mathrm{~V}$; capillary temperature: $270^{\circ} \mathrm{C}$. Selected reaction monitoring was used for detecting DHQ $(161.971 \rightarrow 115.979$, collision energy: $28 \mathrm{~V}$, tube lens: $95 \mathrm{~V}), 2-\mathrm{AA}$ $(136.016 \rightarrow 91.048$, collision energy: $24 \mathrm{~V}$, tube lens: $68 \mathrm{~V})$, HHQ 


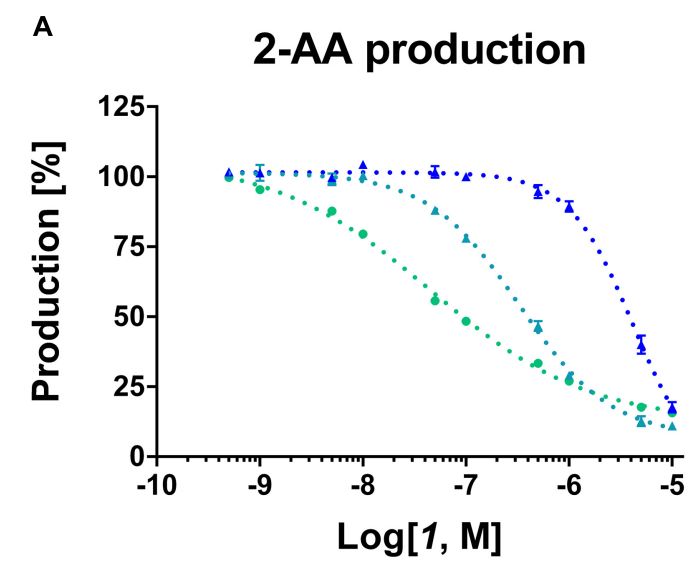

B DHQ production

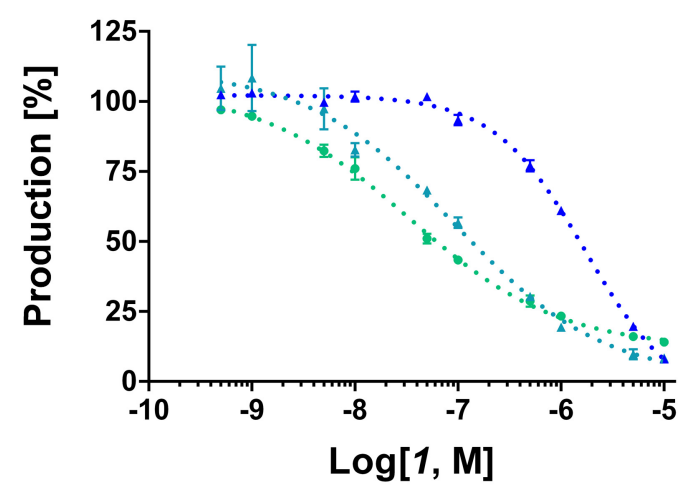

C 2-AA+DHQ production

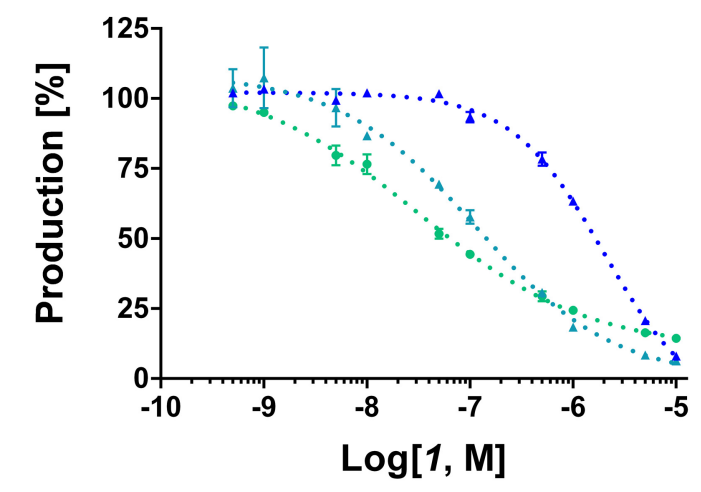

$\triangle \triangle 10 \mu M P Q S$ $\sim 1 \mu M P Q S$

OMM PQS

FIGURE 4 | Dose-response curves of MvfR antagonist 1 on 2-AA (A), $\mathrm{DHQ}$ (B) and overall (C) production in PA14 pqsC mutant with and without external addition of PQS. The " $x$ " axes indicate the logarithm of the concentration of the antagonists in molar units (M). The error bars indicate Standard Error of the Mean

$(244.050 \rightarrow 158.944$; collision energy: $31 \mathrm{~V}$; tube lens: $100 \mathrm{~V})$, HQNO $(260.036 \rightarrow 158.908$; collision energy: $28 \mathrm{~V}$; tube lens: $110 \mathrm{~V})$, PQS (260.048 $\rightarrow$ 174.927; collision energy: $30 \mathrm{~V}$; tube lens: $110 \mathrm{~V}), d_{4}$-HHQ $(248.081 \rightarrow 162.965$; collision energy: $32 \mathrm{~V}$; tube lens: $100 \mathrm{~V})$ and amitriptyline $(278.061 \rightarrow 232.932$; collision energy: $16 \mathrm{~V}$; tube lens: $90 \mathrm{~V}$ ) employing a scan width of 0.010 $\mathrm{m} / z$, a scan time of $0.100 \mathrm{~s}$, and a peak width of 0.70 . Calibration curves were prepared following the same protocol and using PA14 pqsA mutant as matrix without compounds and spiked with known concentrations of analytes and IS after the overnight growth. The assays were repeated at least three times.

\section{pqsA Expression Assay}

The assays were performed following the protocol previously published (Kesarwani et al., 2011) with few modifications. PA14 $w t$ cells transformed with pAC37 were grown overnight in LB with $60 \mu \mathrm{g} / \mathrm{mL}$ of gentamicin, then an aliquot of bacterial culture was centrifuged for $5 \mathrm{~min}$ at $8000 \mathrm{~g}$ at $25^{\circ} \mathrm{C}$ using a 5810R Centrifuge (Eppendorf, United States). The supernatant was discarded, the pellet was resuspended in fresh LB with the same antibiotic, and the bacterial cells were spun down using the previous centrifugation settings. After repeating the wash step a second time, the $\mathrm{OD}_{600 \mathrm{~nm}}$ of the washed cells was measured using a Spectronic Unicam Genesys 10 UV spectrophotometer (Thermo Fisher, United States) in order to prepare a final bacterial suspension with $\mathrm{OD}_{600 \mathrm{~nm}}=0.02$ in LB with $60 \mu \mathrm{g} / \mathrm{mL}$ of gentamicin. Hundred microliters of the prepared culture was poured in each well of a 96-well plate (Corning Inc., Corning, NY, United States) and the compounds were added in triplicates. The final concentration of DMSO was $1 \% \mathrm{v} / \mathrm{v}$. The plates were incubated at $37^{\circ} \mathrm{C}$ under static condition in Infinite F200 plate reader (Tecan Group Ltd, Männedorf, Switzerland) monitoring GFP fluorescence $\left(\lambda_{\text {ex }}=485 \mathrm{~nm} ; \lambda_{\text {em }}=535 \mathrm{~nm}\right)$ and $\mathrm{OD}_{600 \mathrm{~nm}}$ after a short shaking every $15 \mathrm{~min}$. The assays were repeated at least three times.

\section{Persister Cells Assay}

The effects of QSIs on persistence were evaluated following the published protocol (Starkey et al., 2014) with some modifications. After streaking the bacteria on LB agar and overnight incubation at $37^{\circ} \mathrm{C}$, one colony was dispersed in $5 \mathrm{~mL}$ of $\mathrm{LB}$ and the bacteria were grown at $37^{\circ} \mathrm{C}$ up to $\mathrm{OD}_{600 \mathrm{~nm}}=0.5$. Thirty microliters of the culture were transferred into glass tubes with $5 \mathrm{~mL}$ of TSB $1 \%$ $[w / v]$ and incubated overnight at $37^{\circ} \mathrm{C}$ under shaking condition. Then, an aliquot of $P$. aeruginosa culture was centrifuged for $5 \mathrm{~min}$ at $8,000 \times g$ and $25^{\circ} \mathrm{C}$ using a $5810 \mathrm{R}$ Centrifuge (Eppendorf, United States). The supernatant was discarded, the pellet was resuspended in fresh LB, and the bacterial cells were spun down using the previous centrifugation settings. After repeating the wash step a second time, the $\mathrm{OD}_{600 \mathrm{~nm}}$ of the washed cells was measured using a Spectronic Unicam Genesys 10 UV spectrophotometer (Thermo Fisher, United States) in order to prepare a final bacterial suspension with $\mathrm{OD}_{600 \mathrm{~nm}}=0.02$ in $5 \mathrm{~mL}$ of TSB $1 \%[w / v]$ in each glass tube. The target compounds were added in the tubes and the final concentration of DMSO was $0.5 \%[v / v]$. The bacterial suspension was incubated at $37^{\circ} \mathrm{C}$ under shaking condition for $4 \mathrm{~h}$. An aliquot of $100 \mu \mathrm{L}$ of culture from each tube was used for dilution plating on LB agar plates and colony forming units (CFU) quantification (normalizers). Fifty microliters of meropenem $1 \mathrm{mg} / \mathrm{mL}$ were added in each tube and the cultures were incubated at $37^{\circ} \mathrm{C}$ for $24 \mathrm{~h}$ under shaking condition. Aliquots of $600 \mu \mathrm{L}$ of bacterial suspension 
A

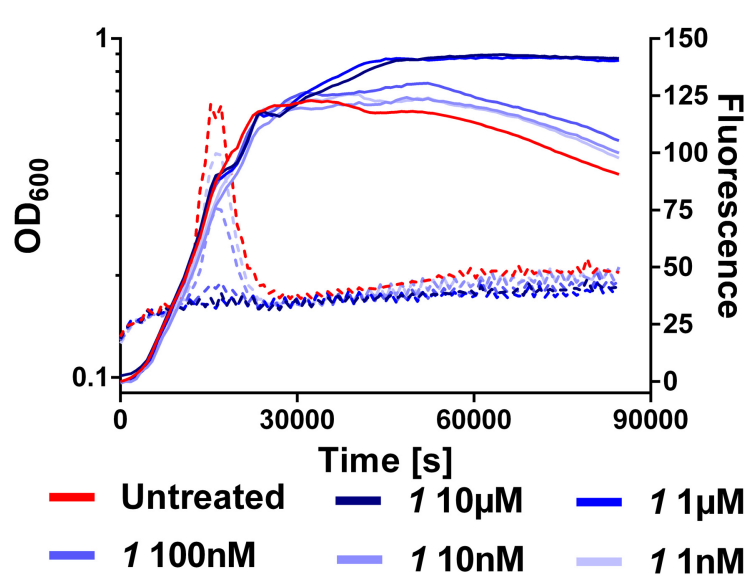

B



FIGURE 5 | Expression of pqsA-GFP ${ }_{\text {ASv }}$ (dotted lines) and growth curves (solid lines) of Pseudomonas aeruginosa treated with 1 (A). IC Po $_{50}$ of 1 (B). The " $x$ " axes indicate the logarithm of the concentration of the antagonists in molar units (M). The error bars indicate Standard Error of the Mean.

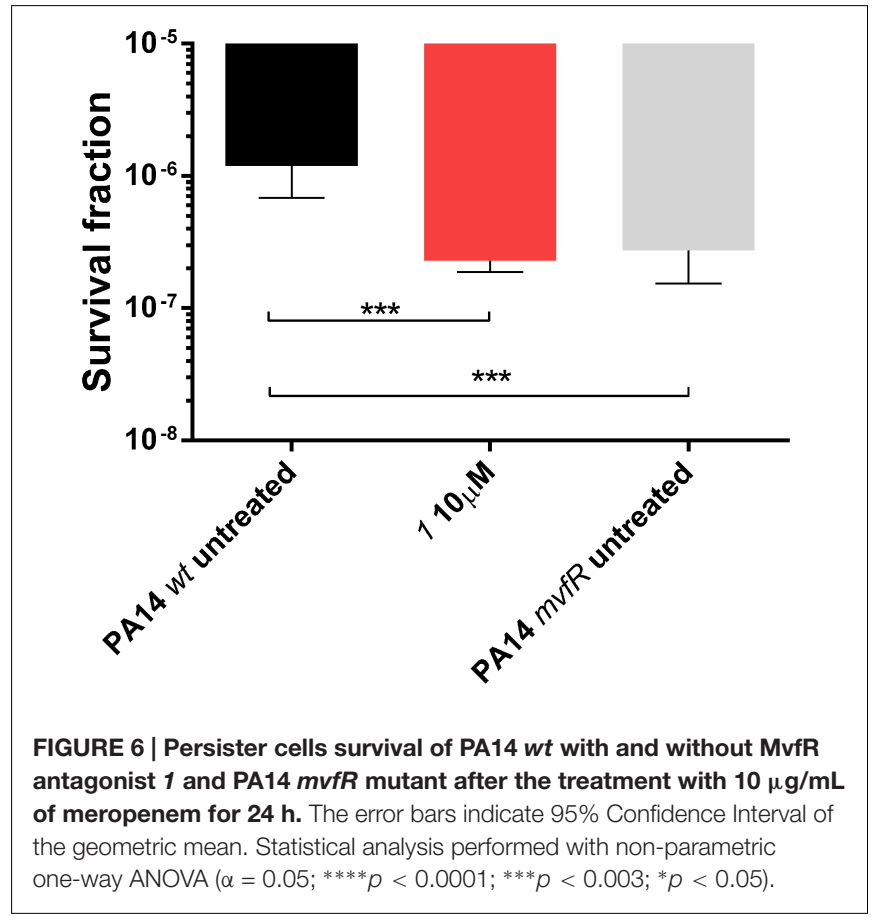

were utilized for dilution plating on LB agar plates and CFU quantification (persisters). The survival fractions were calculated as the ratio of normalizers over persisters. Triplicates per each condition were employed in each assay and the experiments were repeated at least three times.

\section{RESULTS AND DISCUSSION}

The QSIs evaluated in this study were the most potent developed MvfR antagonist 1 (Starkey et al., 2014), and the two PqsBC inhibitors 2 (Starkey et al., 2014) and 3 (this study) shown in Figure 2. As previously published, these compounds were able to inhibit dose-dependently the production of the signal molecules HHQ and PQS in PA14 pqsH mutant and $w t$, respectively. However, a complete profiling of all MvfR-related small molecules was not performed using different concentrations of QSIs. To ensure a convenient analytic procedure, we developed an "all-in-one" method for the simultaneous evaluation of these bacterial metabolites. Following the protocol by Lépine et al. (2003) with some optimizations, a single assay for quantification of the relevant $p q s$-related small molecules was established allowing medium throughput, easy sample processing, low material consumption, without relevant interference between analytes.

\section{Effects of MvfR Antagonists on pqs-Related Small Molecules}

As previously shown (Starkey et al., 2014), the MvfR antagonist 1 was able to reduce dose-dependently the production of the alkyl-quinolones (AQs) and 2-AA in PA14 wt with an $\mathrm{IC}_{50}$ (inhibitor concentration causing half maximum inhibition) of $1.1 \mu \mathrm{M}$ (Table 1; Figure 3A). Interestingly, the sigmoidal curves of each of these metabolites were very steep with a Hill coefficient over 1 giving the idea of a possible exponential effect of the MvfR natural ligands on the pqs regulon expression. Moreover, while DHQ production was inhibited at high concentrations, its biosynthesis was enhanced up to $330 \%$ after incubation of $P$. aeruginosa with $\mathbf{1}$ at a concentration close to the compound' $\mathrm{IC}_{50}$. The basal levels of DHQ were reached at lower doses of the QSI. Nevertheless, 1 was able to reduce dose-dependently the overall biosynthesis of these metabolites with an $\mathrm{IC}_{50}$ of $1.2 \mu \mathrm{M}$.

For confirming this characteristic profile, the compound was also evaluated in PA14 pqsH mutant strain, which does not convert HHQ into PQS. Notably, in a clinical setting, it has been observed that $P$. aeruginosa tends to produce much 
TABLE 3 | Production of pqs signal molecules in PA14 wt after the treatment with PqsBC inhibitors.

\begin{tabular}{|c|c|c|c|c|c|c|}
\hline Compounds & Concentration $[\mu \mathrm{M}]$ & 2-AA [\%] & $\mathrm{DHQ}[\%]$ & HQNO [\%] & $\mathrm{HHQ}+\mathrm{PQS}[\%]$ & Overall [\%] \\
\hline \multirow[t]{4}{*}{2} & 250 & $188 \pm 4$ & $389 \pm 33$ & $198 \pm 12$ & $34 \pm 1$ & $98 \pm 8$ \\
\hline & 50 & $152 \pm 4$ & $249 \pm 15$ & $201 \pm 10$ & $57 \pm 1$ & $101 \pm 5$ \\
\hline & 10 & $111 \pm 8$ & $141 \pm 31$ & $148 \pm 19$ & $81 \pm 6$ & $100 \pm 3$ \\
\hline & 2 & $107 \pm 2$ & $115 \pm 7$ & $128 \pm 1$ & $93 \pm 4$ & $102 \pm 2$ \\
\hline \multirow[t]{4}{*}{3} & 10 & $415 \pm 39$ & $654 \pm 49$ & $218 \pm 16$ & $35 \pm 3$ & $136 \pm 5$ \\
\hline & 1 & $134 \pm 7$ & $131 \pm 12$ & $181 \pm 39$ & $86 \pm 2$ & $100 \pm 5$ \\
\hline & 0.1 & $99 \pm 4$ & $102 \pm 1$ & $103 \pm 2$ & $96 \pm 3$ & $98 \pm 3$ \\
\hline & 0.01 & $99 \pm 4$ & $100 \pm 4$ & $97 \pm 2$ & $103 \pm 5$ & $99 \pm 3$ \\
\hline
\end{tabular}

$100 \%$ is the level of metabolite produced in the untreated PA14 wt. The data are reported with Standard Error of the Mean intervals.
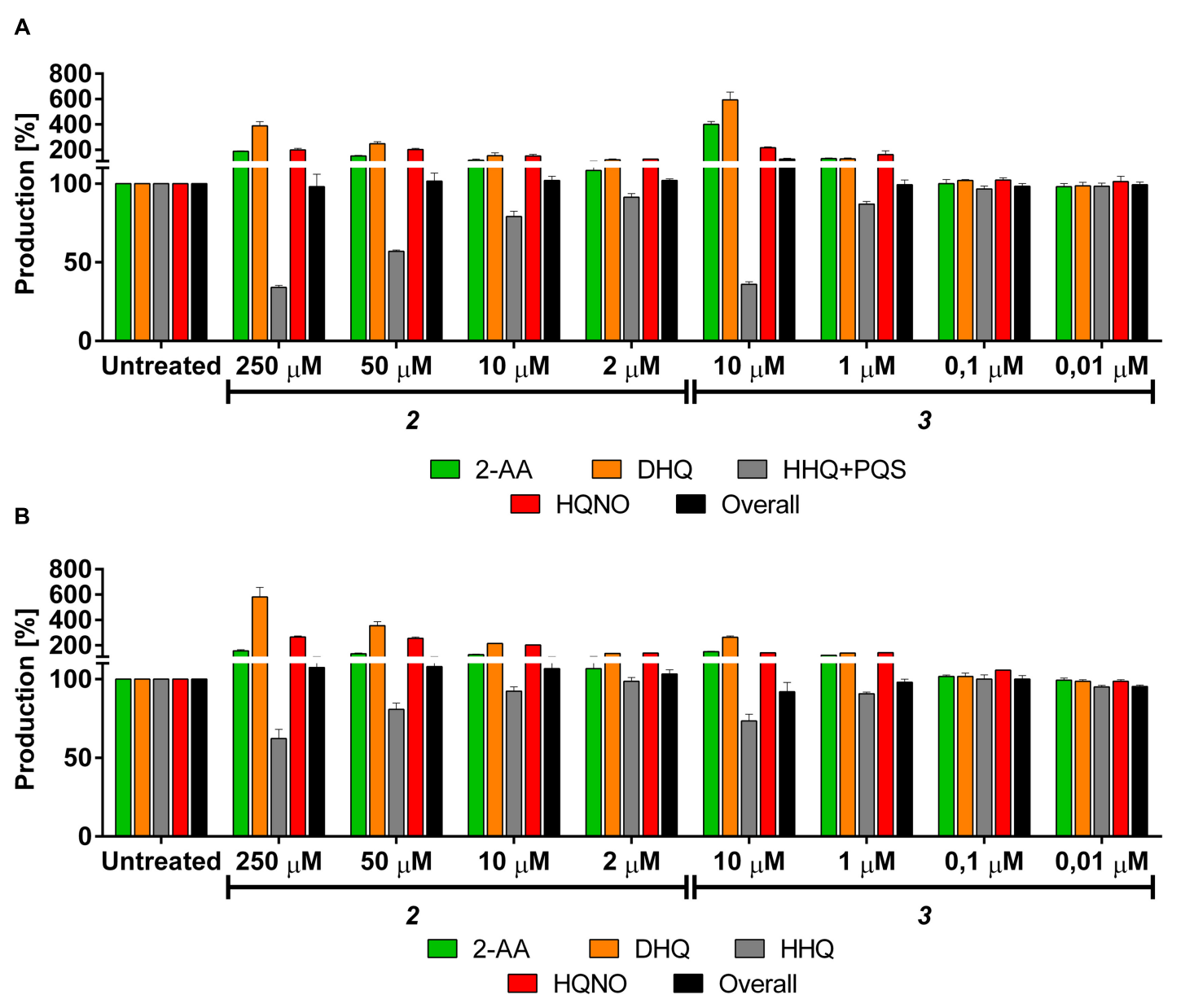

FIGURE 7 | Effects of PqsBC inhibitors on pqs related signal molecules production in PA14 strains. (A) 2 and 3 in PA14 wt. 2 -AA: green. DHQ: orange. PQS + HHQ: gray. HQNO: red. Sum of all anthranilic acid derivatives: black. (B) 2 and 3 in PA14 pqsH mutant. 2-AA: green. DHQ: orange. HHQ: gray. HQNO: red. Sum of all anthranilic acid derivatives: black. The error bars indicate Standard Error of the Mean. Statistical analysis performed with one-way ANOVA ( $\alpha=0.05)$.

more HHQ than the hydroxylated analog (Que et al., 2011). Our experiments with the PQS-deficient pqsH mutant revealed that the QSIs showed similar profiles on the other $p q s$-related molecules production compared to PA14 wt. Indeed, 1 efficiently inhibited AQs and 2-AA production displaying an $\mathrm{IC}_{50}$ of circa $0.30 \mu \mathrm{M}$, and very steep inhibitory curves as in PA14 wt (Table 1; Figure 3B). Likewise, they enhanced DHQ formation up to $300 \%$ at $\mathrm{AQs} \mathrm{IC}_{50}$ concentration. Considering that PQS is more active than HHQ in inducing pqs expression (Xiao et al., 2006), it is not surprising that the MvfR antagonists were more potent in repressing the biosynthesis of pqs-related signal molecules in PA14 pqs $H$ mutant than in $w t$.

The characteristic profiles in $w t$ and $p q s H$ mutant of this class of QSIs, such as the steep inhibitory dose-dependent curves 
TABLE 4 | Production of pqs signal molecules in PA14 pqsH mutant after the treatment with PqsBC inhibitors.

\begin{tabular}{|c|c|c|c|c|c|c|}
\hline Compounds & Concentration $[\mu \mathrm{M}]$ & 2-AA [\%] & $\mathrm{DHQ}[\%]$ & HQNO [\%] & HHQ [\%] & Overall [\%] \\
\hline \multirow[t]{4}{*}{2} & 250 & $157 \pm 8$ & $581 \pm 76$ & $265 \pm 8$ & $62 \pm 6$ & $107 \pm 4$ \\
\hline & 50 & $134 \pm 5$ & $355 \pm 31$ & $255 \pm 10$ & $81 \pm 4$ & $108 \pm 3$ \\
\hline & 10 & $126 \pm 6$ & $215 \pm 3$ & $199 \pm 2$ & $91 \pm 4$ & $104 \pm 4$ \\
\hline & 2 & $102 \pm 2$ & $133 \pm 2$ & $138 \pm 1$ & $97 \pm 2$ & $101 \pm 2$ \\
\hline \multirow[t]{4}{*}{3} & 10 & $150 \pm 3$ & $264 \pm 11$ & $141 \pm 1$ & $73 \pm 4$ & $92 \pm 6$ \\
\hline & 1 & $121 \pm 2$ & $139 \pm 3$ & $143 \pm 2$ & $91 \pm 1$ & $98 \pm 2$ \\
\hline & 0.1 & $102 \pm 1$ & $102 \pm 2$ & $106 \pm 1$ & $100 \pm 3$ & $100 \pm 2$ \\
\hline & 0.01 & $99 \pm 1$ & $99 \pm 1$ & $99 \pm 1$ & $95 \pm 1$ & $95 \pm 1$ \\
\hline
\end{tabular}

$100 \%$ is the level of metabolite produced in the untreated PA14 pqsH mutant. The data are reported with Standard Error of the Mean intervals.

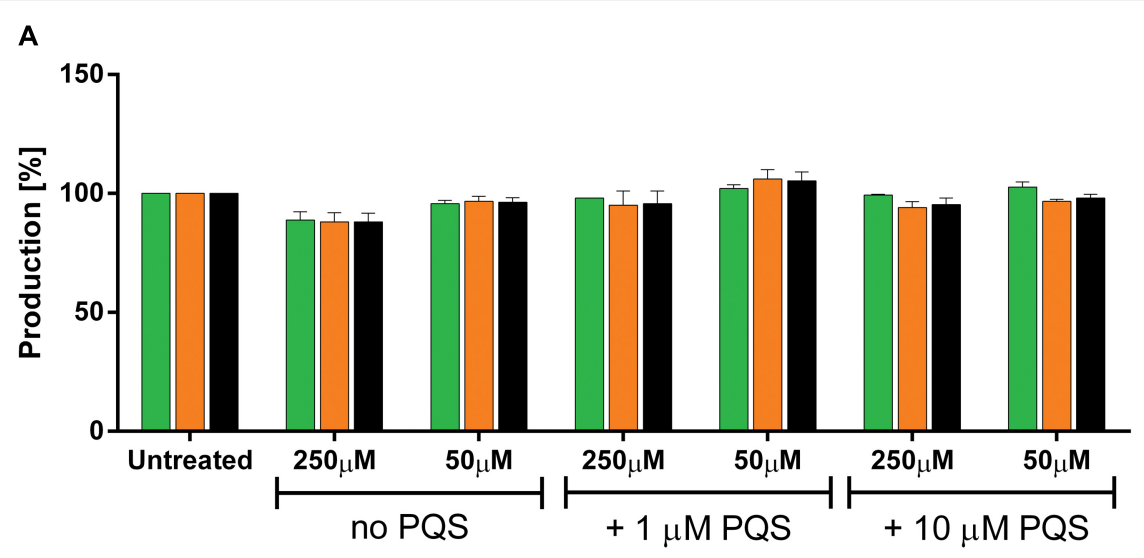

B

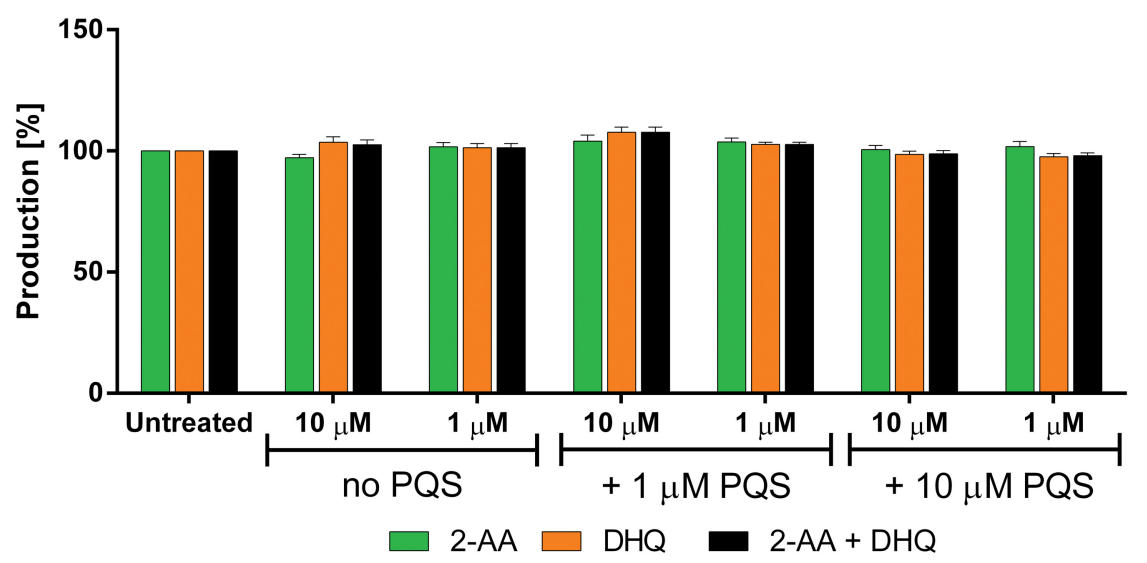

FIGURE 8 | Effects of PqsBC inhibitors on 2-AA and DHQ production in PA14 pqsC mutant. (A) 2 and (B) 3. 2-AA: green. DHQ: orange. Sum of all anthranilic acid derivatives: black. The error bars indicate Standard Error of the Mean. Statistical analysis performed with one-way ANOVA ( $\alpha=0.05)$.

and the overproduction of $\mathrm{DHQ}$ close to the $\mathrm{IC}_{50}$ for $\mathrm{AQ}$ inhibition, suggest the pqs autoloop as a reason. Actually, PQS and HHQ have high activity toward MvfR in the nanomolar range (Xiao et al., 2006; Lu et al., 2014b) and the actual QS signal is amplified through expression of enzymes, which produce again a multitude of additional MvfR natural ligands. Antagonizing the transcriptional regulator would thus have a higher-order effect on the downstream products resulting from pseudo-cooperative effects. Each decrease in signal molecule synthesis achieved by MvfR antagonism would have an additional impact on MvfR deactivation due to less competing autoinducers. In the concentration range close to the antagonist $\mathrm{IC}_{50}$, it would be plausible that the biosynthetic pathway cannot convert the major amount of AA into HHQ and PQS maybe because of a slow kinetic step in the biosynthesis. Considering the low affinity of 2-ABA toward PqsBC (Drees et al., 2016), it is feasible to claim that the slow step is the condensation and cyclization reaction performed by PqsBC. This would lead to an accumulation 

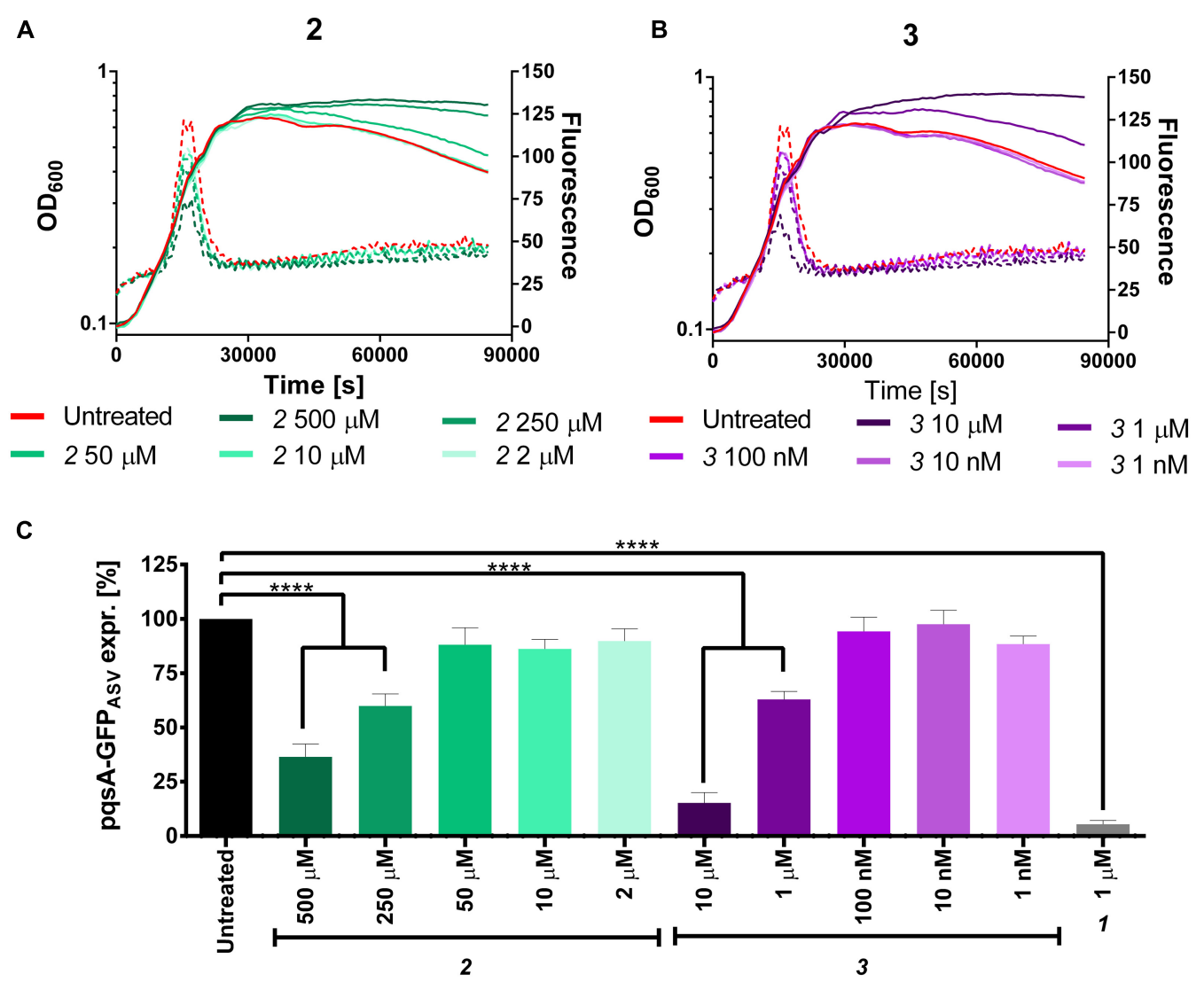

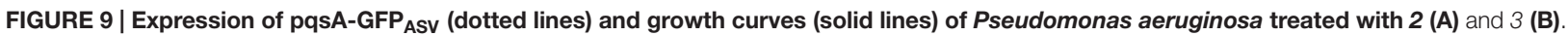
(C) Percentage of pqsA-GFP ASv expression after PqsBC inhibitors addition. The error bars indicate Standard Error of the Mean. Statistical analysis performed with one-way ANOVA $(\alpha=0.05 ; * * * * p<0.0001)$.

of the reactive intermediate 2 -ABA that quickly cyclizes into DHQ.

For confirming these hypotheses, the compound was evaluated in PA14 pqsC mutant, which synthesizes only 2-AA and $\mathrm{DHQ}$, in an experimental setup with and without exogenous addition of the signal molecule PQS. Since these bacteria do not produce any MvfR natural ligands, the $p q s$ autoloop is consequently absent and it was possible to control its expression with external administration of PQS. Exogenous addition of the quinolone at 1 and $10 \mu \mathrm{M}$ reduced the potency of the QSIs on MvfR-related small molecules synthesis of circa one and two orders of magnitude, respectively, and increased the steepness of the inhibitory curves (Table 2; Figure 4). Actually, the $\mathrm{IC}_{50}$ of 1 on 2-AA production worsened from $50 \mathrm{nM}$ (without PQS) over $0.3 \mu \mathrm{M}$ (with $1 \mu \mathrm{M}$ PQS) to $4.2 \mu \mathrm{M}$ (10 $\mu \mathrm{M}$ PQS). In addition, the concentration of compound needed to reduce DHQ production to $50 \%$ shifted from $30 \mathrm{nM}$ (without PQS) over $90 \mathrm{nM}(1 \mu \mathrm{M}$ PQS) to $1.7 \mu \mathrm{M}(10 \mu \mathrm{M}$ PQS). Consequently, the overall effect of the QSI on the production of pqs-related molecules within the PqsADE biosynthetic pipeline present in the $p q s C$ mutant was also affected by PQS administration. Hence, an increase of $\mathrm{IC}_{50 s}$ from $30 \mathrm{nM}$ (no PQS) over $0.11 \mu \mathrm{M}(1 \mu \mathrm{M}$ PQS) to $2.0 \mu \mathrm{M}$ (10 $\mu \mathrm{M}$ PQS) was observed.
These findings confirmed that the presence of PQS in the bacterial culture plays an important role in controlling the biosynthesis of pqs-related molecules. Indeed, when the natural ligand is present, the efficiency of the compounds was strongly reduced as the shifts in $\mathrm{IC}_{50}$ confirmed. In addition, the increased steepness of the inhibitory curves after addition of PQS to the PA14 pqsC mutant culture displayed that, in case of a small reduction in concentration of QSIs, the MvfR-related compounds were quickly restored to the basal production level.

Furthermore, the expression levels of $p q s A$ were monitored under MvfR antagonist treatment using the PA14 wt transformed with the plasmid carrying the construct $p q s A-\mathrm{GFP}_{\mathrm{ASV}}$ (Yang et al., 2007). As previously reported (Kesarwani et al., 2011), the kinetic studies showed that the expression of the $p q s A$ promoter present in the $p q s A-\mathrm{GFP}_{\mathrm{ASV}}$ plasmid occurred at the exponential phase of bacterial growth as it was analyzed for the genomic pqs operon (Déziel et al., 2004).As formerly evaluated (Starkey et al., 2014), 1 efficiently inhibited the promoter expression displaying an $\mathrm{IC}_{50}$ of $16 \mathrm{nM}$ (Figure 5).

Combining these findings with the results in PA14 wt and $p q s H$ mutant indirectly confirmed that the action of autoinducers HHQ and PQS within this positive feedback loop is the 




explanation for the pronounced steepness of the sigmoidal dose-response curves for 2-AA, HHQ, PQS, and HQNO levels. The fact that down-regulation of the pqs operon results in an initial increase in DHQ, while the other investigated components show the expected sigmoidal dose-response curves, suggests that either 2-ABA-CoA or 2-ABA accumulate in this scenario, which can be spontaneously degraded to the shunt product. From published studies on the whole PQS biosynthesis cassette, it can be assumed that the reactions mediated by the PqsAD-cascade proceed quickly enough also at lowered enzyme concentrations to enable sufficient 2-ABA-CoA production. These initial steps should be more dependent on the cellular availability of the substrates AA and malonyl-CoA. The hydrolysis catalyzed by PqsE can also be performed by housekeeping thioesterases like TesB. But, an analysis of a pqsE transposon mutant by Drees and Fetzner (2015) regarding the profile of PQS-related molecules showed increased DHQ levels. We corroborated and extended the data on this strain with the established pqs-related small molecule quantification method (Supplementary Figure S1). As expected for this strain, 2-AA levels are reduced while DHQ concentration is dramatically increased hinting at accumulation of the reactive intermediate 2-ABA-CoA. Finally, insufficient action of PqsBC and, thus, accumulation of 2-ABA should lead to both increased DHQ and 2-AA levels.

Hence, the gathered information suggests that through the MvfR-antagonist-induced down-regulation of the $p q s$ operon an initial accumulation of 2-ABA-CoA rather than 2-ABA takes place (as in the $p q s E$ mutant). Interestingly, it has been shown that the condensation reaction mediated by PqsBC should be the rate-limiting step in this biosynthetic cascade
(Drees and Fetzner, 2015). Thus, we consider it rather surprising that we observe a metabolite profile, that hints more toward the role of 2-ABA-CoA upon partial inactivation of the pqs operon.

Nevertheless, at very low levels of MvfR activity, DHQ is finally reduced as the diminished PqsAD cascade ceases to operate efficiently resulting in the observed metabolite profiles.

Among the pqs-related molecules produced by this pathogen, 2-AA was shown to be a key factor for switching the infection from acute to chronic state favoring the formation of persister cells (Kesarwani et al., 2011; Que et al., 2013). Actually, high levels of this QS compound enhanced the genesis of antibiotic tolerant cells within the population reducing the killing activity of antibiotics. Considering the high efficiency of the MvfR antagonists in reducing 2-AA (Starkey et al., 2014), these QSIs were evaluated in their capability to modify the survival fraction of the pathogen after treatment with meropenem. A dose of $10 \mu \mathrm{M}$ of 1 reduced significantly persisters development in PA14 $w t$ from $1.2 \times 10^{-6}$ of the untreated control to $2.3 \times 10^{-7}$ reaching the same levels of the 2-AA non-producing strain PA14 $m v f R$ mutant, that is $2.7 \times 10^{-7}$ (Figure 6) as previously demonstrated (Starkey et al., 2014). These findings corroborated the importance of suppressing the biosynthesis of this carbonyl compound via blockage of the transcriptional regulator for achieving a more efficient antibiotic therapy against $P$. aeruginosa infections.

\section{Effects of PqsBC Inhibitors on mvfR-Related Small Molecules}

The PqsBC inhibitors 2 and 3 reduced the production of the MvfR natural ligands in PA14 wt down to 34 and 35\%, respectively, at their highest concentration (Table 3; Figure 7A). As expected (vide supra), the levels of 2-AA and DHQ strongly increased after the treatment with such inhibitors up to 188 and $389 \%$ after incubation with $250 \mu \mathrm{M}$ of 2 and 415 and $654 \%$ with $10 \mu \mathrm{M}$ of 3. Surprisingly, the synthesis of HQNO was also enhanced up to two times compared to the untreated bacteria. Interestingly, the overall production of the AQ signal molecules was not significantly affected. Reducing the concentration of these QSIs led to a reduced inhibitory activity on HHQ and PQS production and a relapse of 2-AA, DHQ and HQNO to the respective basal levels.

For confirming this characteristic profile, the compounds were also evaluated in PA14 pqsH mutant and similar results were obtained compared to the isogenic $w t$ (Table 4; Figure 7B). Here, 2 and 3 reduced at their highest dosage the formation of HHQ down to 62 and 73\%, respectively. Moreover, the production of 2-AA, DHQ, and HQNO was increased by 157,581 , and $265 \%$ after treatment with $250 \mu \mathrm{M}$ of 2 and 150, 264, and 141\% after incubation with $10 \mu \mathrm{M}$ of 3 compared to the untreated control. In addition, as in PA14 wt, the overall amount of the MvfR-related compounds was not affected by the addition of these QSIs.

Additionally, 2 and 3 were examined in PA14 pqsC mutant for confirming their target selectivity. Both compounds turned out to be inactive in reducing 2-AA and DHQ production independently from the concentration of PQS added into the 
culture (Figure 8) supporting the in vitro characterization of the two inhibitors (unpublished results; Starkey et al., 2014).

Considering their efficiency in reducing the production of the MvfR natural ligands in PA14 wt and pqsH mutant, these inhibitors were analyzed in the pqsA-GFP $\mathrm{ASV}$ construct for monitoring their potential ability for reducing the expression of the pqs operon. Actually, 2 and $\mathbf{3}$ reduced the operon transcription down to 36 and 15\%, respectively, at their highest dosage (Figure 9). However, it is not clear in this setting why no autolysis is observed despite the presence of HQNO, which is responsible for the programmed cell autolysis observed in liquid cultures of $P$. aeruginosa (Hazan et al., 2016).

Taking into consideration the obtained results, the effects of the PqsBC inhibitors on the MvfR natural ligands, 2-AA, and DHQ production fitted to the expected behavior of blocking the hetero-dimer PqsBC. Actually, its inhibition would lead to a reduced conversion of the reactive 2-ABA into HHQ with consequently reduced expression of the pqs operon. The excess of this intermediate would be consequently transformed more into the stable molecules 2-AA and DHQ, conversions that do not require PqsBC. Notably, as previously reported, PqsBC inhibitor 3 efficiently reduced HQNO, HHQ and PQS production at a concentration of around $90 \mu \mathrm{M}$ (Starkey et al., 2014). Hence, full PqsBC inhibition occurs at concentrations higher than the ones used in the present study, which hints to a similar concentration dependency as observed for DHQ levels upon application of MvfR antagonists. According to the current model of HQNO synthesis, 2-ABA is $N$-oxidized by the oxidase PqsL into its hydroxylamine form, 2-HABA. We assume that this moiety of the intermediate should be a more reactive nucleophile (Ningst et al., 2012) than the amine of 2-ABA or a better substrate and that, consequently, the reaction of condensation and cyclization performed by the hetero-dimer proceeds faster. Moreover, it is plausible to expect that the enzyme complex has a different affinity toward the amine and the hydroxylamine intermediates and, due to this fact, the inhibitors would have also different activities in blocking both reactions. Based on the obtained results, this hypothesis would reasonably explain the overproduction of HQNO after incubation of PA14 with PqsBC inhibitors. But, we do not exclude that an additional, yet unknown bypass reaction toward HQNO might cause this unexpected observation. In addition, the analysis of the overall amount of the pqs-related small molecules revealed that these QSIs modify only the ratio of the QS compounds without affecting the total content.

As described above, QS profiles observed for PqsBC inhibitors were different from those determined for the MvfR antagonist. In particular, the inductive effects on 2-AA production drew our attention. Hence, we examined 2 and 3 regarding their ability to affect the development persister cells (as reported in Starkey et al., 2014). We found that only 2 significantly enhanced the persistence phenotype of PA14 wt increasing the survival fraction from $1.2 \times 10^{-6}$ (untreated) up to $4.6 \times 10^{-6}$ cells. This corresponds to the levels of PA14 wt treated with 2-AA as well as the untreated PA14 pqsBC mutant showing persister rates of $7.2 \times 10^{-6}$ and $4.1 \times 10^{-6}$, respectively (Figure 10). These findings confirmed that targeting PqsBC led to survival of the bacteria in the presence of antibiotic. This might ultimately result in a reduced efficiency of antibiotic therapy.

\section{CONCLUSION}

The profiling of $P$. aeruginosa MvfR antagonist and PqsBC inhibitors emphasized the importance in selecting the target for the development of new anti-infectives, and confirmed that MvfR is an excellent target for a global AQ and 2-AA inhibition. Actually, antagonizing the transcriptional regulator led to an efficient inhibition of the pqs operon expression and, consequently, of the $m v f R$-small molecules production. As it was found an overproduction of the toxic DHQ at AQs $\mathrm{IC}_{50}$ doses, the current study highlighted that an efficient therapy would be obtained only choosing a concentration of QSI capable to fully suppress the MvfR activity and, in the end, the biosynthetic pathway. The PqsBC inhibitors showed to be less efficient in reducing the MvfR natural ligands synthesis and, moreover, lead to an increased production of 2-AA and DHQ. Actually, they mainly affected the distribution of QS molecules generated within a bacterial population without modifying the overall production. The importance of reducing 2-AA production through MvfR antagonism is critical in preventing the formation of antibiotic tolerant persister cells. In case of PqsBC inhibitors, a combination with other QSIs (e.g., PqsA, PqsD, or MvfR) might still be a valid route toward the use of a novel anti-infective approach.

\section{AUTHOR CONTRIBUTIONS}

GA: Performed experiments and wrote the paper. CM and JE: Supervised LC-MS experiments and wrote the paper. DM and LR: Supervised cellular experiments. RH and ME: Supervised experiments and wrote the paper.

\section{FUNDING}

The research was supported by the Helmholtz Centrum for Infection Research, Massachusetts General Hospital and by "GradUS global" - promoted by the German Academic Exchange Service (DAAD) and funded by the Federal Ministry of Education and Research (BMBF). This work was supported in part by NIH R33AI105902 to LGR.

\section{ACKNOWLEDGMENT}

We would like to thank Prof. Dr. Susanne Häussler for kindly supplying Pseudomonas aeruginosa PA14 wt and its isogenic $p q s A, p q s C, p q s E$ and $p q s H$ mutants.

\section{SUPPLEMENTARY MATERIAL}

The Supplementary Material for this article can be found online at: http://journal.frontiersin.org/article/10.3389/fmicb. 2017.00924/full\#supplementary-material 


\section{REFERENCES}

Aloush, V., Navon-Venezia, S., Seigman-Igra, Y., Cabili, S., and Carmeli, Y. (2006). Multidrug-resistant Pseudomonas aeruginosa: risk factors and clinical impact. Antimicrob. Agents Chemother. 50, 43-48. doi: 10.1128/AAC.50.1.43-48.2006

Déziel, E., Lépine, F., Milot, S., He, J., Mindrinos, M. N., Tompkins, R. G., et al. (2004). Analysis of Pseudomonas aeruginosa 4-hydroxy-2alkylquinolines (HAQs) reveals a role for 4-hydroxy-2-heptylquinoline in cellto-cell communication. PNAS 101, 1339-1344. doi: 10.1073/pnas.0307694100

Drees, S. L., and Fetzner, S. (2015). PqsE of Pseudomonas aeruginosa acts as pathway-specific thioesterase in the biosynthesis of alkylquinolone signaling molecules. Chem. Biol. 22, 611-618. doi: 10.1016/j.chembiol.2015.04.012

Drees, S. L., Li, C., Prasetya, F., Saleem, M., Dreveny, I., Williams, P., et al. (2016). PqsBC, a condensing enzyme in the biosynthesis of the Pseudomonas aeruginosa quinolone signal: crystal structure, inhibition, and reaction mechanism. J. Biol. Chem. 291, 6610-6624. doi: 10.1074/jbc.M115. 708453

Dulcey, C. E., Dekimpe, V., Fauvelle, D.-A., Milot, S., Groleau, M.-C., Doucet, N., et al. (2013). The end of an old hypothesis: the Pseudomonas signaling molecules 4-hydroxy-2-alkylquinolines derive from fatty acids, not 3-ketofatty acids. Chem. Biol. 20, 1481-1491. doi: 10.1016/j.chembiol.2013.09.021

Gómez, I. M., and Prince, A. (2007). Opportunistic infections in lung disease: Pseudomonas infections in cystic fibrosis. Curr. Opin. Pharmacol. 7, 244-251. doi: 10.1016/j.coph.2006.12.005

Gruber, J. D., Chen, W., Parnham, S., Beauchesne, K., Moeller, P., Flume, P. A., et al. (2016). The role of 2,4-dihydroxyquinoline (DHQ) in Pseudomonas aeruginosa pathogenicity. PeerJ. 4:e1495. doi: 10.7717/peerj.1495

Hancock, R. E. W., and Speert, D. P. (2000). Antibiotic resistance in Pseudomonas aeruginosa: mechanisms and impact on treatment. Drug Resist. Updat. 3, 247-255. doi: 10.1054/drup.2000.0152

Hazan, R., Que, Y.-A., Maura, D., Strobel, B., Majcherczyk, P. A., Hopper, L. R., et al. (2016). Auto poisoning of the respiratory chain by a quorum-sensingregulated molecule favors biofilm formation and antibiotic tolerance. Curr. Biol. 26, 195-206. doi: 10.1016/j.cub.2015.11.056

Hurley, M. N., Cámara, M., and Smyth, A. R. (2012). Novel approaches to the treatment of Pseudomonas aeruginosa infections in cystic fibrosis. Eur. Respir. J. 40, 1014-1023. doi: 10.1183/09031936.00042012

Kesarwani, M., Hazan, R., He, J., Que, Y.-A., Apidianakis, Y., Lesic, B., et al. (2011). A quorum sensing regulated small volatile molecule reduces acute virulence and promotes chronic infection phenotypes. PLoS Pathog. 7:8. doi: 10.1371/journal. ppat. 1002192

Lee, J., and Zhang, L. (2015). The hierarchy quorum sensing network in Pseudomonas aeruginosa. Protein Cell 6, 26-41. doi: 10.1007/s13238-0140100-x

Lépine, F., Deziel, E., Milot, S., and Rahme, L. G. (2003). A stable isotope dilution assay for the quantification of the Pseudomonas quinolone signal in Pseudomonas aeruginosa cultures. Biochim. Biophys. Acta 1622, 36-41. doi: 10.1016/S0304-4165(03)00103-X

Lewis, K. (2010). Persister cells. Annu. Rev. Microbiol. 64, 357-372. doi: 10.1146/ annurev.micro.112408.134306

Lu, C., Kirsch, B., Maurer, C. K., de Jong, J. C., Braunshausen, A., Steinbach, A., et al. (2014a). Optimization of anti-virulence PqsR antagonists regarding aqueous solubility and biological properties resulting in new insights in structure-activity relationship. Eur. J. Med. Chem. 79, 173-183. doi: 10.1016/ j.ejmech.2014.04.016

Lu, C., Kirsch, B., Zimmer, C., de Jong, J. C., Henn, C., Maurer, C. K., et al. (2012). Discovery of antagonists of PqsR, a key player in 2-alkyl-4-quinolonedependent quorum sensing in Pseudomonas aeruginosa. Chem. Biol. 19, 381-390. doi: 10.1016/j.chembiol.2012.01.015

Lu, C., Maurer, C. K., Kirsch, B., Steinbach, A., and Hartmann, R. W. (2014b). Overcoming the unexpected functional inversion of a PqsR antagonist in Pseudomonas aeruginosa: an in vivo potent antivirulence agent targeting pqs quorum sensing. Angew. Chem. Int. Ed. 53, 1109-1112. doi: 10.1002/anie. 201307547
Maurer, C. K., Steinbach, A., and Hartmann, R. W. (2013). Development and validation of a UHPLC-MS/MS procedure for quantification of the Pseudomonas Quinolone Signal in bacterial culture after acetylation for characterization of new quorum sensing inhibitors. J. Pharm. Biomed. Anal. 86, 127-134. doi: 10.1016/j.jpba.2013.07.047

Mulcahy, L. R., Burns, J. L., Lory, S., and Lewis, K. (2010). Emergence of Pseudomonas aeruginosa strains producing high levels of persister cells in patients with cystic fibrosis. J. Bacteriol. 192, 6191-6199. doi: 10.1128/JB. 01651-09

Ningst, T. A., Antipova, A., and Mayr, H. (2012). Nucleophilic reactivities of hydrazines and amines: the futile search for the $\alpha$-effect in hydrazine reactivities. J. Org. Chem. 77, 8142-8155. doi: 10.1021/jo301497g

Que, Y.-A., Hazan, R., Ryan, C. M., Milot, S., Lépine, F., Lydon, M., et al. (2011). Production of Pseudomonas aeruginosa intercellular small signal molecules in human burn wounds. J. Pathog. 2011:549302. doi: 10.4061/2011/ 549302

Que, Y.-A., Hazan, R., Strobel, B., Maura, D., He, J., Kesarwani, M., et al. (2013). A quorum sensing small volatile molecule promotes antibiotic tolerance in bacteria. PLOS ONE 8:e80140. doi: 10.1371/journal.pone.0080140

Rahme, L. G., Lépine, F., Starkey, M., and Lesic-Arsic, B. (2012). Antibiotic Tolerance Inhibitor. US 61/445,448.

Schertzer, J. W., Brown, S. A., and Whiteley, M. (2010). Oxygen levels rapidly modulate Pseudomonas aeruginosa social behaviours via substrate limitation of PqsH. Mol. Microbiol. 77, 1527-1538. doi: 10.1111/j.1365-2958.2010. 07303.x

Starkey, M., Lépine, F., Maura, D., Bandyopadhaya, A., Lesic, B., He, J., et al. (2014). Identification of anti-virulence compounds that disrupt Quorum-Sensing regulated acute and persistent pathogenicity. PLoS Pathog. 10:e1004321. doi: 10.1371/journal.ppat.1004321

Storz, M. P., Allegretta, G., Kirsch, B., Empting, M., and Hartmann, R. W. (2014). From in vitro to in cellulo: structure-activity relationship of (2-nitrophenyl)methanol derivatives as inhibitor of $\mathrm{PqsD}$ in Pseudomonas aeruginosa. Org. Biomol. Chem. 12, 6094-6104. doi: 10.1039/C4OB00707G

Tredget, E. E., Shankowsky, H. A., Rennie, R., Burrell, R. E., and Logsetty, S. (2004). Pseudomonas infections in the thermally injured patient. Burns 30, 3-26. doi: 10.1016/j.burns.2003.08.007

Xiao, G., Déziel, E., He, J., Lépine, F., Lesic, B., Castonguay, M.-H., et al. (2006). MvfR, a key Pseudomonas aeruginosa pathogenicity LTTR-class regulatory protein, has dual ligands. Mol. Microbiol. 62, 1689-1699. doi: 10.1111/j.13652958.2006.05462.x

Yang, L., Barken, K. B., Skindersoe, M., Christensen, A. B., Givskov, M., and TolerNielsen, T. (2007). Effects of iron on DNA release and biofilm development by Pseudomonas aeruginosa. Microbiology 153, 1318-1328. doi: 10.1099/mic.0. 2006/004911-0

Zender, M., Klein, T., Henn, C., Kirsch, B., Maurer, C. K., Kail, D., et al. (2013). Discovery and biophysical characterization of 2-amino-oxadiazoles as novel antagonists of PqsR, an important regulator of Pseudomonas aeruginosa virulence. J. Med. Chem. 56, 6761-6774. doi: 10.1021/jm400830r

Zhang, Y. M., Frank, M. W., Zhu, K., Mayasundari, A., and Rock, C. O. (2008). $\mathrm{PqsD}$ is responsible for the synthesis of 2,4-dihydroxyquinoline, an extracellular metabolite produced by Pseudomonas aeruginosa. J. Biol. Chem. 283, 2878828794. doi: 10.1074/jbc.M804555200

Conflict of Interest Statement: The authors declare that the research was conducted in the absence of any commercial or financial relationships that could be construed as a potential conflict of interest.

Copyright (c) 2017 Allegretta, Maurer, Eberhard, Maura, Hartmann, Rahme and Empting. This is an open-access article distributed under the terms of the Creative Commons Attribution License (CC BY). The use, distribution or reproduction in other forums is permitted, provided the original author(s) or licensor are credited and that the original publication in this journal is cited, in accordance with accepted academic practice. No use, distribution or reproduction is permitted which does not comply with these terms. 